\title{
Traumatic pancreatic transection - Case reports
}

\section{Laudari U' ID $ه$, Pradhan R², Rajak A ${ }^{3}$ iD, Parajuli A ${ }^{4}$ iD, Maharjan DK ${ }^{5}$ iD, Thapa PB ${ }^{6}$}

'Uttam Laudari, Department of Surgery, Hospital for Advanced Medicine and Surgery, Kathmandu, Nepal; ${ }^{2}$ Rosi Pradhan, Department of Anesthesiology, KIST Medical College, Lalitpur, Nepal; ${ }^{3}$ Ashik Rajak, Department of Surgery; ${ }^{4}$ Anuj Parajuli, Lecturer, Department of Surgery; ${ }^{5}$ Dhiresh Kumar Maharjan, Assistant Professor, Department of Surgery; ${ }^{6}$ Prabin Bikram Thapa, Professor, Department of Surgery, Kathmandu Medical College Teaching Hospital, Kathmandu, Nepal.

\begin{abstract}
Pancreatic injury following blunt trauma abdomen is presentation with significant morbidity and mortality. Our first case here is the American Association for the Surgery of Trauma Organ Injury Scale (AAST-OIS) Grade V pancreaticoduodenal injuries following penetrating injury managed with pancreaticoduodenectomy and the second case is blunt trauma to the abdomen with pancreatic tail transection which is American Association for the Surgery of Trauma Organ Injury Scale (AAST-OIS) Grade III that was managed with Endoscopic Retrograde Cholangiopancreaticography (ERCP) and pancreatic duct stenting. The outcome depends on early diagnosis and needs a high index of suspicion due to subtle clinical and radiological findings initially. Hemodynamically unstable patients may have other associated visceral and vascular injuries, and damage control strategy will be a suitable option. Same sitting resection procedures like pancreaticoduodenectomy are rarely performed. Pancreatic injuries can often be managed non-operatively with percutaneous drainage and pancreatic duct stenting.
\end{abstract}

Key words: Conservative treatment; Pancreaticoduodenectomy; Pancreatic trauma; Radiological drainage endoscopic treatment.

\section{INTRODUCTION}

Dancreatic injury accounts for $0.2 \%$ to $12 \%$ of all - abdominal trauma, and injury by gunshot or stab accounts for $20 \%$ to $30 \%$ of all patients with penetrating injuries ${ }^{1,2}$. These injuries are common in children and young adults as they have a sparse amount of protective fat around pancreas than adults ${ }^{2}$. Pancreatic body,

Access this article online

Website: www.jkmc.com.np

DOI: https://doi.org/10.3126/jkmc.v9i3.36415

\section{HOW TO CITE}

Laudari U, Pradhan R, Rajak A, Parajuli A, Maharjan DK, Thapa PB.

Traumatic pancreatic transection - Case reports. J Kathmandu Med Coll. 2020;9(3):165-8.

Address for correspondence

\section{Dr. Uttam Laudari}

Department of Surgery

Hospital for Advanced Medicine and Surgery

Kathmandu, Nepal

E-mail:youttam@hotmail.com

Copyright @ 2020 Journal of Kathmandu Medical College (JKMC)

ISSN: 2019-1785 (Print), 2091-1793 (Online)

(i) (S) This work is licensed under a Creative Commons Attribution-Non Commercial 4.0 International License. head and tail are common sites in order of injury and are mostly associated with co-existent other injuries (60\% are duodenopancreatic lesions, while $90 \%$ involve at least one other abdominal organ)2. Our first case was a young thin built male who had a penetrating injury which was associated with duodenopancreatic injury and was successfully managed with same sitting pancreaticoduodenectomy whereas the second case was a thin built young female who presented with pancreatic tail injury and was managed with the percutaneous and endoscopic approach. Both of the cases had subtle clinical and radiological findings on presentation; one of which was diagnosed intra-operatively and the other was confirmed on serial imaging due to clinical deterioration.

\section{CASE REPORTS}

\section{Case 1}

A twenty-four-year-old male presented with an alleged history of fall from a two-storey building within one hour of accident. He sustained injury over the lower abdomen and left thigh accompanied by bleeding from injured sites. On examination, the patient was hemodynamically stable, with normal mentation and neurological status. The patient had a $3 \times 3 \mathrm{~cm}$ puncture wound in the right lumbar region, admitting two fingers with fascial dehiscence and breached peritoneum 
with no pulsatile bleeding. Abdominal examination revealed tender epigastrium with no other features of peritonitis. In addition, he also had two small puncture wounds in his left thigh which were of no significance. Laboratory values revealed WBC of $16200 / \mathrm{mm}^{3}$, hemoglobin $14.4 \mathrm{gm} / \mathrm{dl}$, creatinine $110 \mathrm{uMol} / \mathrm{l}$, plasma electrolytes and liver function test within normal limits. Focused assessment with sonography in trauma (FAST scan) was negative. Contrast enhanced computerized tomography (CECT) of the abdomen trauma series showed pneumoperitoneum and an exploratory laparotomy was planned (Figure 1). Intra-operatively there was near complete transection of duodenum near duodenojejunal flexure, hemoperitoneum and retroperitoneal hematoma in zone I and zone II. There was complete transection of the neck of pancreas (AASTOIS Grade V injury) with saponification around mesentery and penetrating unhealthy wound in the right lumbar region (Figure 1). Pancreas texture was soft, with small duct and the common bile duct (CBD) was approximately $5 \mathrm{~mm}$. The patient was further managed with Whipple pancreaticoduodenectomy. Intra-operatively one-pint whole blood was transfused. The patient had Grade B pancreatic fistula ${ }^{3}$, had wound infection and was discharged on the eight post-operative day with the drain removed on the $21^{\text {st }}$ post-operative period and was doing fine on the $90^{\text {th }}$ day follow up.

\section{Case 2}

A twenty-year-old female with an alleged history of blunt abdominal trauma following a landslide presented with complaints of abdominal pain. The patient was hemodynamically stable and was shifted to the Intensive Care Unit (ICU) for monitoring. Laboratory investigations showed WBC of $15000 / \mathrm{mm}^{3}$, hemoglobin $13.5 \mathrm{gm} / \mathrm{dl}$, CPK-MB $1432 \mathrm{U} / \mathrm{L}$, serum lipase $680 \mathrm{U} / \mathrm{l}$, total bilirubin 0.5 $\mathrm{mg} / \mathrm{dl}$, direct bilirubin $1.7 \mathrm{mg} / \mathrm{dl}$, aspartate transaminase $165 \mathrm{U} / \mathrm{L}$ and alanine transaminase values of $211 \mathrm{U} / \mathrm{L}$. CECT abdomen revealed AAST-OIS Grade I splenic and Grade II liver laceration. Pancreatic contusion at tail and peripancreatic tail fat stranding was seen with mild free fluid in peritoneum (Figure 2). There was no fall in hematocrit and no features of peritonitis, but her abdominal pain was in increasing trend. She had occasional fever with daily increase in WBC and had persistent unwellness until the eight day of admission. There was no significant increase in peritoneal fluid in daily FAST scan. Therefore, CECT abdomen was done which revealed loss of pancreatic tissue at tail (AAST-OIS Grade III), progression of pancreatitis with acute pancreatic fluid collection (APFC) of size $16 \times 10 \times 6.5 \mathrm{~cm}$, CT severity index of 6 and hence a percutaneous pigtail was inserted which drained approximately one litre of pancreatic ascites (Drain amylase - $67600 \mathrm{U} / \mathrm{L})$. The patient was discharged on the $13^{\text {th }}$ day of admission with pigtail in-situ which was
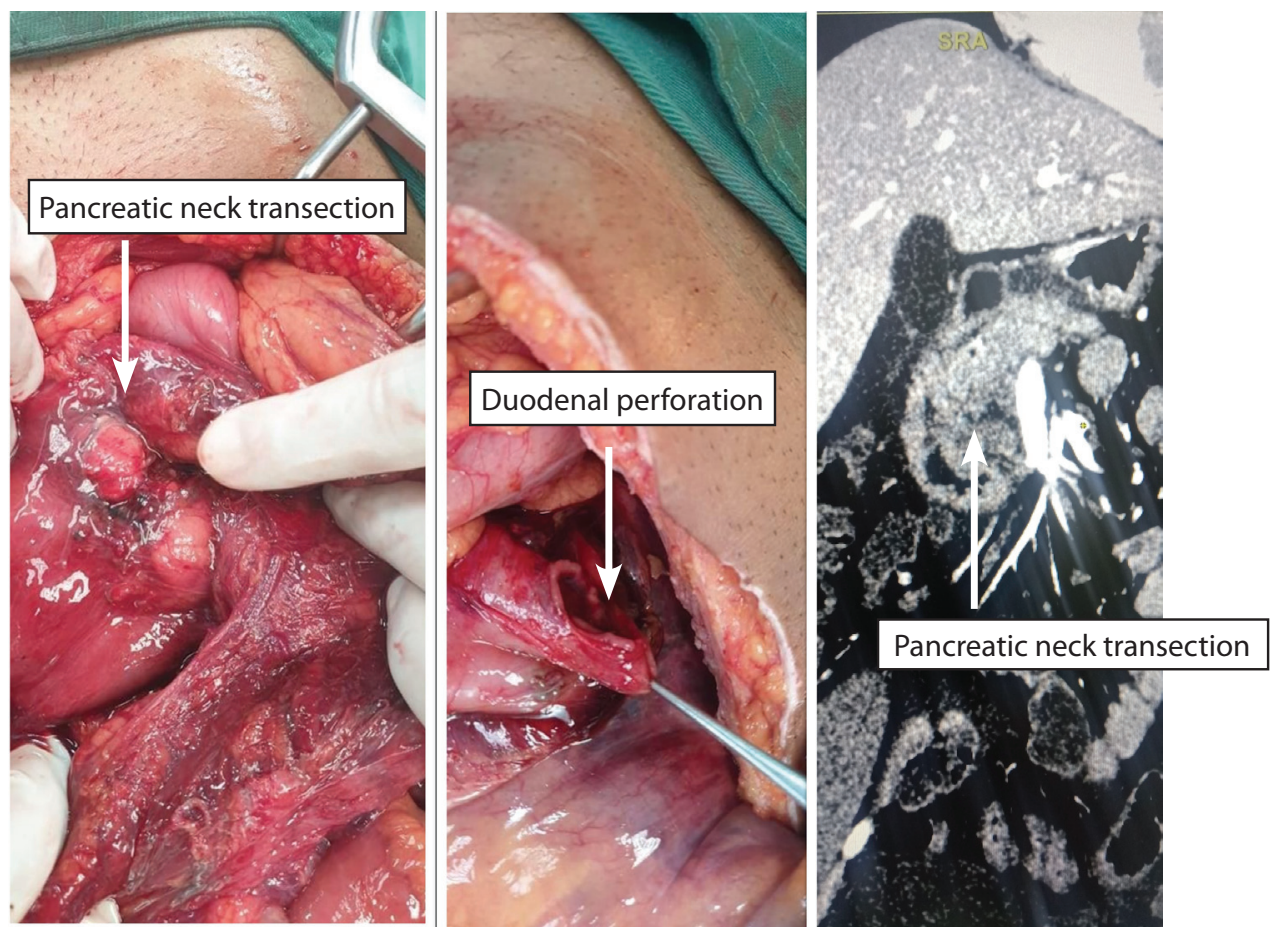

Figure 1: Intra-operative pictures showing pancreatic neck transection and associated duodenal perforation at duodenojejunal flexure and CECT abdomen of same patient showing pancreatic injury. 
draining approximately 150 millilitres (ml) daily. After four weeks she still had persistent pancreatic fistula draining approximately 80 to $100 \mathrm{ml}$ daily, and presented to the emergency department with abdominal pain. Magnetic Resonance Cholangiopancreaticography (MRCP) was done which showed similar findings of previous CECT with suspected proximal pancreatic duct fistula with peri-pancreatic fluid collection $(12 \times 8.8 \times 3.3 \mathrm{~cm})$ (Figure
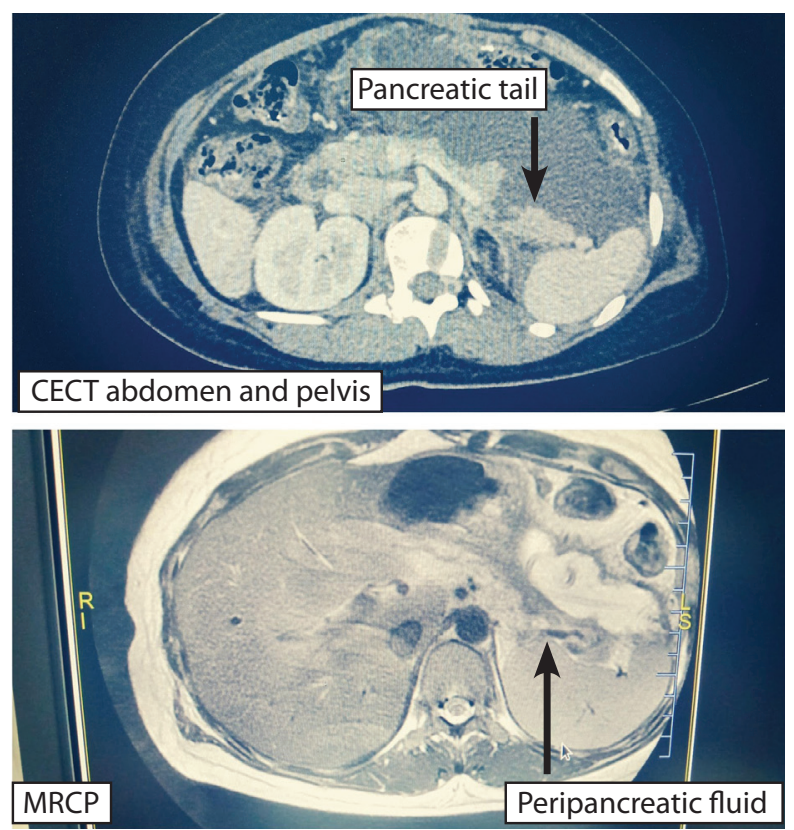

Figure 2: CECT abdomen of Case 2 showing pancreatic tail transection with associated acute pancreatic fluid collection and MRCP of same patient after a month, showing pancreatic duct fistula with peri-pancreatic fluid collection.

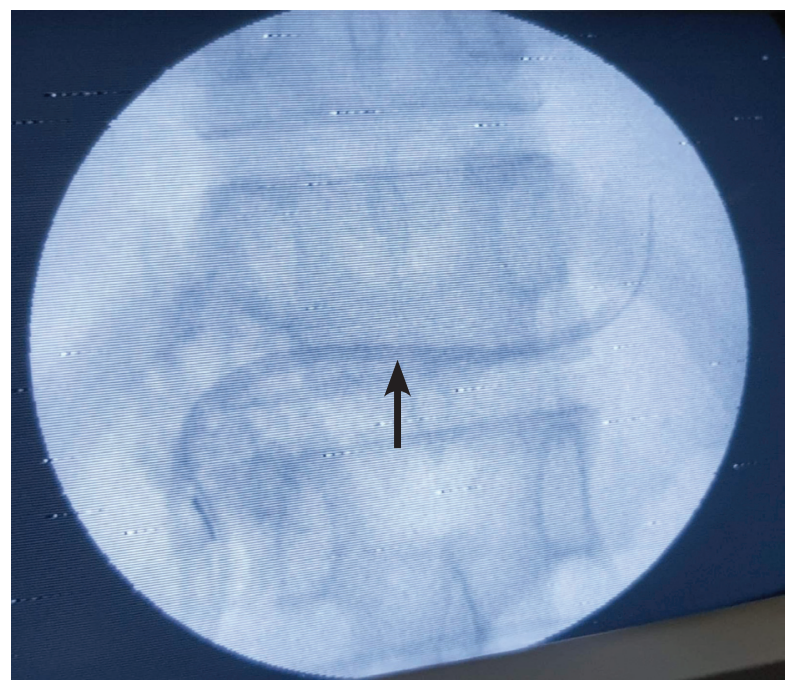

Figure 3: ERCP showing pancreatic duct stenting (arrow) in Case 2.
2). Hence, ERCP and pancreatic duct stenting was done with $7 \mathrm{Fr} 10 \mathrm{~cm}$ long straight biliary stent with note of its tip proximal to cut-off area (Figure 3), after which the amount of drain decreased. She was discharge post ERCP and stenting with pigtail drainage in situ. The drain was removed six weeks after the procedure and the patient was doing fine on the $90^{\text {th }}$ day follow up.

\section{DISCUSSION}

Our first case was abdominal penetrating injury managed with emergency pancreaticoduodenectomy for transection of pancreatic head and associated duodenal perforation whereas the second case was pancreatic tail transection following blunt abdominal trauma managed with endoscopic manoeuvre.

The clinical triad of upper abdominal pain, leukocytosis and raised amylase may not always be evident during the first 24 hours or even later in pancreaticoduodenal injuries ${ }^{2}$. After initial investigation following general principles of Advanced trauma life support (ATLS), patients should undergo FAST scan and CECT although both of these tests have low sensitivity of $40 \%$ to $50 \%$ for pancreatic injury 4,5 . In such cases, CECT abdomen may show hard signs such as parenchymal lacerations and soft signs like peripancreatic fluid. A finding of $50 \%$ parenchymal transection has high risk of ductal disruption. In our first case, associated pancreatic injury was missed in CECT pre-operatively whereas in the second case the patient had fat stranding around tail of pancreas with pancreatitis, splenic injury and liver laceration. Due to persistent unwellness of patients, persistent abdominal pain and progressive systemic inflammatory response syndrome (SIRS) in eighth day CECT abdomen was repeated where pancreatic tail injury was more delineated with large acute pancreatic fluid collection compressing stomach anteriorly. The AASTOIS is a universally used grading system for pancreatic injury but it does not take associated duodenal injury into account. Lucas score and Frey \& Wardell have also included associated duodenal injury which is more useful for practical management, but is not being used universally ${ }^{6}$.

Hemodynamically unstable patients are shifted straight away to the operating room, where a diagnosis can be confirmed. Damage control laparotomy followed by staged surgery can also be done $e^{4}$. Our first case was shifted to the operating room for pneumoperitoneum, and was intra-operatively found to have pancreaticoduodenal injury. For stable patients, MRCP becomes the investigation of choice to better delineate the pancreatic 
duct status. ERCP may show contrast extravasation from side or main duct injuries. ERCP has added advantage of stenting the duct in the same sitting as an initial temporary method in stable patients ${ }^{4}$. Our second case was initially managed with percutaneous pigtail drainage for pancreatic fluid collection, followed by MRCP, ERCP and pancreatic duct stenting for persistent pancreatic fistula. Serum amylase and lipase may be mildly raised within six hours of injury; however, these findings are not specific as they are raised in other intra-abdominal injuries too. Amylase level detected in diagnostic peritoneal lavage is more sensitive than serum amylase ${ }^{2}$.

Grade I and II injuries can be managed conservatively as long as there is no involvement of pancreatic duct ${ }^{7}$. Endoscopic management via ERCP and pancreatic duct stenting can be done as in our second case which can prevent major laparotomy, resectional procedures, shorten hospital stay and treatment of late onset complications $s^{4,8}$. For grade III or IV pancreatic injury identified either on CT scan or during surgery, operative or resectional treatment is recommended. In Grade V injury, there is still debate regarding routine performance of pancreaticoduodenectomy due to very high mortality and morbidity'. Resection procedure such as pancreaticoduodenectomy is rarely done in same sitting outside high volume centers. Outcomes

\section{REFERENCES}

1. Ho VP, Patel NJ, Bokhari F, Madbak FG, Hambley $\mathrm{JE}$, Yon JR, et al. Management of adult pancreatic injuries: A practice management guideline from the Eastern Association for the Surgery of Trauma. J Trauma Acute Care Surg. 2017;82(1):185-99. [Full Text | PubMed]

2. Debi U, Kaur R, Prasad KK, Sinha SK, Sinha A, Singh K. Pancreatic trauma: A concise review. World J Gastroenterol. 2013;19(47):9003-11. [Full Text | PubMed]

3. Bassi C, Marchegiani G, Dervenis C, Sarr M, Abu Hilal M, Adham M, et al. The 2016 update of the International Study Group (ISGPS) definition and grading of postoperative pancreatic fistula: 11 Years After. Surg. 2017;161(3):584-91. [Full Text | PubMed | DOI]

4. Søreide K, Weiser TG, Parks RW. Clinical update on management of pancreatic trauma. HPB [Internet]. 2018;20(12):1099-108. [Full Text | PubMed] of pancreaticoduodenal injury depends on the grade of injury, amount of blood loss, duration of shock; number, extent and magnitude of associated injury; efficacy of resuscitation and speed and quality of surgical intervention ${ }^{9,10}$. It is appropriate to undergo initial surgical drainage until the patient is well resuscitated for definitive surgical procedure and timely referral to center with pancreatic surgery expertise for better outcome ${ }^{4}$.

\section{CONCLUSION}

Higher grade pancreatic injuries need a competent and experienced multi-disciplinary team. Endoscopic treatment for higher grade injury can be valuable to patients who are hemodynamically stable with distal injuries in an acute setting whereas resectional procedures like pancreaticoduodenectomy in trauma setting need mature judgement, skilled evaluation and intraoperative assistance of a hepatopancreaticobiliary surgeon.

\section{ACKNOWLEDGMENT}

Dr. Murari Raj Upreti, Dr. Suraj Lamichhane, Dr. Manindra Manandhar, Dr. Hem Paneru, Dr. Sabin Koirala and Dr. Kamal Raj Subedi.

\section{Conflict of interest: None Source(s) of support: None}

5. Sharma AK. Management of Pancreaticoduodenal Injuries. Indian J Surg. 2012;74(1):35-9. [Full Text | PubMed]

6. Lucas CE. Diagnosis and treatment of pancreatic and duodenal injury. Surg Clin North Am [Internet]. 1977;57(1):49-65. [Full Text | PubMed]

7. Menahem B, Lim C, Lahat E, Salloum C, Osseis $M$, Lacaze $L$, et al. Conservative and surgical management of pancreatic trauma in adult patients. HepatoBiliary Surg Nutr. 2016;5(6):470-7. [PubMed]

8. Petrone P, Moral Álvarez S, González Pérez M, Ceballos Esparragón J, Marini CP. Management of pancreatic trauma: a literature review. Cirugía Española. 2017;95(3):123-30. [Full Text | PubMed]

9. Krige JE, Nicol AJ, Navsaria PH. Emergency pancreatoduodenectomy for complex injuries of the pancreas and duodenum. Hpb [Internet]. 2014;16(11):1043-9. [Full Text | PubMed]

10. Heuer M, Hussmann B, Lefering R, Taeger G, Kaiser GM, Paul A, et al. Pancreatic injury in 284 patients with severe abdominal trauma: Outcome, course, and treatment algorithm. Langenbeck's Arch Surg. 2011;396(7):1067-76. [Full Text | PubMed] 